\title{
Calibration of an Indexing Table Using a High Precision Angle Comparator
}

\author{
Ming Huang ${ }^{1,2}$, PinKuan $\mathrm{Liu}^{1}$, Yangqiu Xia ${ }^{2,3}$, Qiu Hu², Mengyang $\mathrm{Li}^{2}$, Qiang Tang ${ }^{2,3}$ \\ ${ }^{1}$ State Key Laboratory of Mechanical System and Vibration, Shanghai Jiao Tong University, Shanghai, China \\ ${ }^{2}$ Institute of Machinery Manufacturing Technology, China Academy of Engineering Physics, Mianyang, China \\ ${ }^{3}$ National Machine Tool Production Quality Supervision Testing Center(Sichuan), Mianyang, China
}

\begin{abstract}
The calibration of indexing table is important to ensure high accuracy and reliability in angle metrology. In this paper, we present calibrations of an indexing table and uncertainty analysis to evaluate the performance of the high precision angle comparator. The configuration of the angle comparator is explained. In the calibration, a complete closure method is applied to obtain the angle comparator's position error and the indexing table's angular deviation simultaneously based on the least square method. The position error of the angle comparator is evaluated to be about 0.08 ". The calibration result of an indexing table is compatible with that of the manufacturer and National Institute of Metrology of China within the expanded uncertainty of calibration, $0.04 "$.
\end{abstract}

\section{Introduction}

Accurate angle measure technologies are required in various precision engineering applications, e.g., manufacturing, robotics [1,2]. Indexing tables generate accurate angular positions, and their calibrations are important to ensure high accuracy and reliability in angle metrology [3].

A multi-tooth indexing table is a circular indexing table based on the mechanical indexing principle. A multi-tooth indexing table is composed of two face gears with the same diameter, tooth shape and tooth number, and a similar structure of a multi-tooth face clutch.

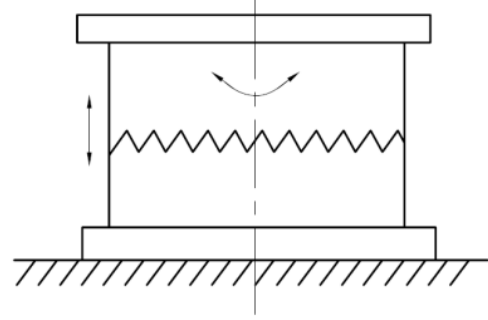

Figure 1. Indexing table.

As shown in Figure 1, when the upper gear disc along the axial direction rises to a certain height, gear discs remove meshing. After a turn of several teeth relative to the fixed lower gear disc, the upper disc moves down again and meshes with the lower gear disc with a certain axial pressure, thus generating. a certain angle. Obviously, a multi-tooth indexing table can generate or calibrate constant angles. Since all the teeth are involved in meshing positioning, the average effect and elastic overconstrained principle of multi-tooth meshing make indexing table positioning accuracy much higher than gear disc machining accuracy. 0.1" accuracy can be reached. Because on any concentric circle, the thickness and slot width remain the same, the indexing accuracy is not affected by the direction of rotation. The repeatability is also ensured by the centrality of teeth.

The angle calibration has been widely studied by many researchers. Yandayan [4] has calibrated highresolution electronic autocollimators with demanded low uncertainties using single reading head angle encoders. At the Physikalisch-Technische Bundesanstalt (PTB) [5], high-resolution electronic autocollimators have been calibrated with the aid of the WMT 220 angle comparator to ensure their direct traceability to the SI unit of plane angle, the radian (rad). The Korea Research Institute of Standards and Science (KRISS) also developed a high precision angle generator [6, 7], and Jong-Ahn Kim [8] calibrated several angle artifacts and instruments using the high precision angle generator to demonstrate the performance of the angle generator. Huang [9] has compared angle interferometer with angle encoder during calibration of autocollimator.

In this paper, we analyze the performance of the angle comparator, and adopt the complete closure method to calibrate an indexing table. In the calibration of the indexing table, the performance of the angle comparator was evaluated and the measurement uncertainty was analyzed.

\section{High precision angle comparator}

The high precision angle comparator consists of a radial aerostatic bearing, a thrust aerostatic bearing, four ultrasonic motors, a precision angle encoder, and a 
controller. The thrust aerostatic bearing is preloaded with vacuum to stabilize the air clearance and improve the stiffness. The thrust stiffness is $400 \mathrm{~N} / \mu \mathrm{m}$ and the load capacity is $80 \mathrm{~kg}$. There are four ultrasonic motors placed around the rotating plate. The motor tips drive the plate running at fine resolution less than $0.002 "$. The radial run-out of the comparator is less than $0.05 \mu \mathrm{m}$.

Figure 3 shows the block diagram of the angle comparator control system, which is composed of a SPiiPlus universal drive interface (UDIhp4422N01N, $\mathrm{ACS}^{\mathrm{TM}}$ ), a PC based real-time motion controller (SPiiPlusSC HP0800004BAY5A, ACSTM), a motor driver (AB2, Nanomotion ${ }^{\mathrm{TM}}$ ), four Ultrasonic motors (HR2, Nanomotion ${ }^{\mathrm{TM}}$ ) and a feedback encoder with two encoder heads (ERP880, Heidenhain ${ }^{\mathrm{TM}}$ ). The four ultrasonic motors are connected in parallel to synchronize the motion of each motor. The position data of the two heads are averaged to obtain the readout of the angle generator by using a virtual gantry control mode. The sampling and update rates of the servo loop are both 20 $\mathrm{kHz}$.

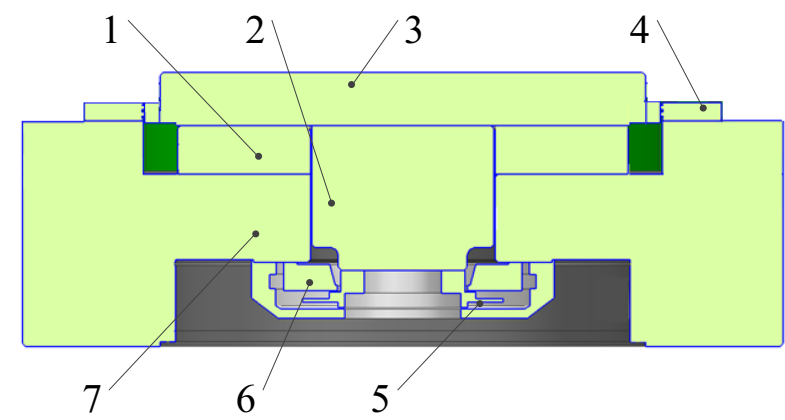

Figure 2. Schematic view of the angle comparator ZT 320: (1) air-bearing casing; (2) air-bearing rotor; (3) table; (4) ultrasonic motor; (5) divided circle; (6) reading heads; (7) supports.

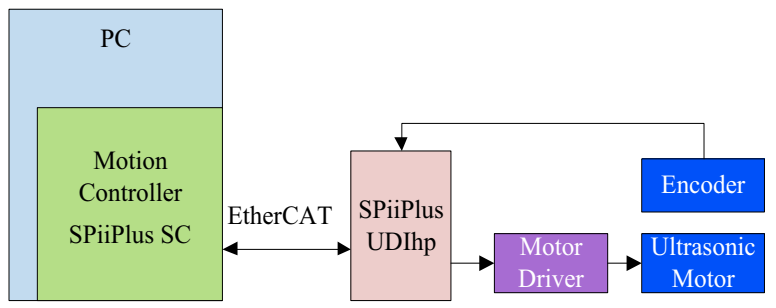

Figure 3. Block diagram of the controller of the angle comparator.

\section{Calibration of indexing tables with the angle comparator}

Figure 4 shows the measurement arrangement for the calibration of a 1440 precision indexing table $( \pm 0.1$ " of arc accuracy) on the angle comparator. The measurement system is composed of the optical polygon, the indexing table, the angle comparator, and the autocollimator. The autocollimator (TA US 300-57 from TRIOPTICS GMBH) has a measurement range of $3000^{\prime \prime} \times 2200^{\prime \prime}$ and a resolution of $0.005 "$. According to manufacturer specifications, the accuracy amounts to \pm 0.05 " over any 10 " range and \pm 0.10 " over any 20 " range.

The axes of the angle comparator, the indexing table and the polygon should coincide with each other. Centred with respect to the comparator's axis of rotation, the autocollimator is standing on an adjustable plate on the granite base. With the aid of the adjustable plate, the autocollimator is aligned such that the horizontal measuring axis and the optical axis of the autocollimator lie in the comparator's measuring plane, and aimed at the reflecting surface of the polygon. The polygon and the indexing table lie in the comparator's axis of rotation to avoid any lateral displacements of the mirror in the autocollimator aperture. The flatness deviations of the mirror have nearly no influence on the calibration result. The air path of $20 \mathrm{~mm}$ between the autocollimator and the reflecting surface of the polygon does not need to be specially shielded since the constant temperature and the laminar airflow in the clean room laboratory do not exert any significant disturbing influences.

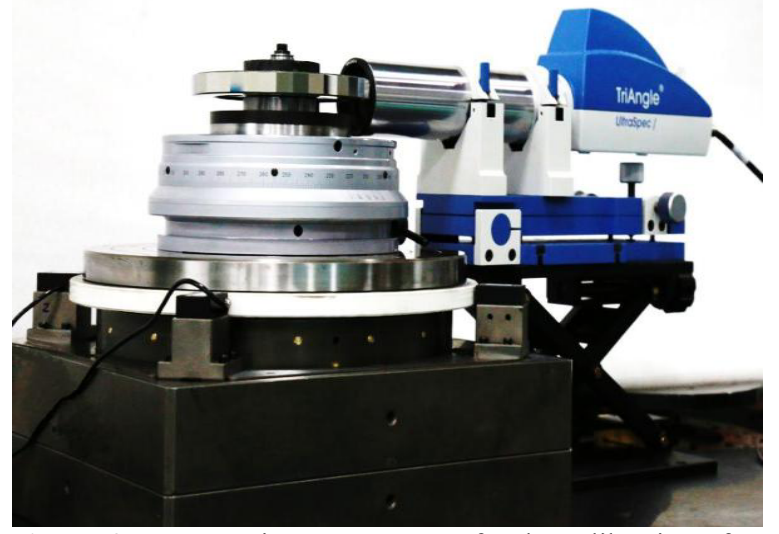

Figure 4. Measuring arrangement for the calibration of an indexing table on the angle comparator.

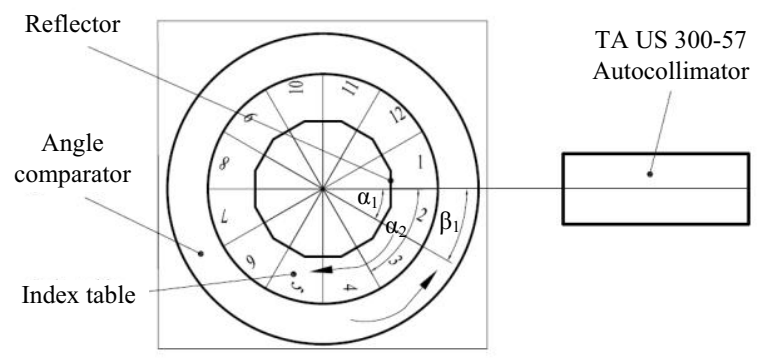

Figure 5. The diagram of the complete closure method.

Calibration is performed by the complete closure method (Figure 5). The method is that a group of constant angles, which are proper, not precise but must be stable, are chosen and used to compare with the measured angles in sequence, respectively and independently in a closed circle. And it can eliminate the influence of the angular deviation of the constant angles and calculate the measured angular deviation at equal weight based on the least square method. If we choose the same angles as the indexing table from the angle comparator, the angular deviations of the indexing table and the comparator will be obtained simultaneously. 
During calibration, by rotating the angle comparator clockwise and the indexing table counter-clockwise, by the same nominal angle $\left(30^{\circ}\right)$, the autocollimator is always aimed at the same reflecting surface of the polygon, the angular deviation will be measured by the autocollimator. The measurement circle will be repeated 12 times in one cycle. The indexing table will be shifted by one nominal angle relative to the angle comparator. After that, the indexing table will rotate from next angle step counter-clockwise to ensure that the autocollimator is always aimed at the identical reflecting surface of the polygon.

The method will eliminate the influence of the constant angle's deviation on the measurement and give both the angle comparator's position error and the indexing table's angular deviation simultaneously based on the least square method.

$\mathrm{n}$ (number of the constant angles) is 12 , and the nominal values of the constant angles can be expressed as

$$
\varphi_{i}=i \frac{360^{\circ}}{n}, \quad i=1,2,3, \cdots, n-1
$$

When the constant angles have been defined, according to the definition of error, the autocollimator reading value can be expressed by

$$
\theta_{i}=\alpha_{i}-\beta_{i}=\left(\varphi_{i}+\delta \alpha_{i}\right)-\left(\varphi_{i}+\delta \beta_{i}\right)=\delta \alpha_{i}-\delta \beta_{i}
$$

where $\alpha_{i}$ is the rotation angle of the indexing table, $\beta_{i}$ is the angle of the angle comparator rotation, $\delta \alpha_{i}$ is the deviation of angle $\alpha_{i}$ to the constant angles, $\delta \beta_{i}$ is the deviation of angle $\beta_{i}$ to the constant angles.

For the first measurement cycle, the indexing table will be rotated in sequence to compare with the angle comparator rotation and a group of measurement numbers will be obtained:

$$
\left\{\begin{array}{c}
\left(\delta \alpha_{1}-\delta \alpha_{0}\right)-\delta \beta_{1}=\theta_{0,1} \\
\left(\delta \alpha_{2}-\delta \alpha_{0}\right)-\delta \beta_{2}=\theta_{0,2} \\
\vdots \\
\left(\delta \alpha_{11}-\delta \alpha_{0}\right)-\delta \beta_{11}=\theta_{0,11}
\end{array}\right.
$$

For the i-th measurement circle,

$$
\left\{\begin{array}{c}
\left(\delta \alpha_{i \bmod 12}-\delta \alpha_{i-1}\right)-\delta \beta_{1}=\theta_{i-1, i \bmod 12} \\
\left(\delta \alpha_{(i+1) \bmod 12}-\delta \alpha_{i-1}\right)-\delta \beta_{2}=\theta_{i-1,(i+1) \bmod 12} \\
\vdots \\
\left(\delta \alpha_{(i+10) \bmod 12}-\delta \alpha_{i-1}\right)-\delta \beta_{11}=\theta_{i-1,(i+10) \bmod 12}
\end{array}\right.
$$

For the last measurement circle,

$$
\left\{\begin{array}{c}
\left(\delta \alpha_{0}-\delta \alpha_{11}\right)-\delta \beta_{1}=\theta_{11,0} \\
\left(\delta \alpha_{1}-\delta \alpha_{11}\right)-\delta \beta_{2}=\theta_{11,1} \\
\vdots \\
\left(\delta \alpha_{10}-\delta \alpha_{11}\right)-\delta \beta_{11}=\theta_{11,10}
\end{array}\right.
$$

Combine the above equations,

$$
\left\{\begin{array}{c}
\sum_{i=1}^{12}\left(\delta \alpha_{i \bmod 12}-\delta \alpha_{i-1}\right)-12 \delta \beta_{1}=\sum_{i=1}^{12} \theta_{i-1, i \bmod 12} \\
\sum_{i=1}^{12}\left(\delta \alpha_{(i+1) \bmod 12}-\delta \alpha_{i-1}\right)-12 \delta \beta_{2}=\sum_{i=1}^{12} \theta_{i-1,(i+1) \bmod 12} \\
\vdots \\
\sum_{i=1}^{12}\left(\delta \alpha_{(i+10) \bmod 12}-\delta \alpha_{i-1}\right)-12 \delta \beta_{11}=\sum_{i=1}^{12} \theta_{i-1,(i+10) \bmod 12}
\end{array}\right.
$$

and

$$
\delta \beta_{j}=-\frac{1}{12} \sum_{i=1}^{12} \theta_{i-1,(i+j-1) \bmod 12}, \quad j=1,2, \cdots, 11
$$

There are totally 132 measurements and 11 constant angles will be obtained. Through Eq. (7), all the deviation of the constant angles generated by the indexing table will be calculated out. In every measurement round, the error equations containing the residuals $\mathrm{V}$ can be listed :

$\left\{\begin{array}{c}\left(\delta \alpha_{i \bmod 12}-\delta \alpha_{i-1}\right)-\delta \beta_{1}-\theta_{i-1, i \bmod 12}=V_{i-1, i \bmod 12} \\ \left(\delta \alpha_{(i+1) \bmod 12}-\delta \alpha_{i-1}\right)-\delta \beta_{2}-\theta_{i-1,(i+1) \bmod 12}=V_{i-1,(i+1) \bmod 12} \\ \vdots \\ \left(\delta \alpha_{(i+10) \bmod 12}-\delta \alpha_{i-1}\right)-\delta \beta_{11}-\theta_{i-1,(i+10) \bmod 12}=V_{i-1,(i+9) \bmod 12}\end{array}\right.$

11 unknowns $\delta \alpha_{i}$ are to be solved from the above 132 equations, and it is obviously indefinite. In order to get the best value of the unknown $\delta \alpha_{i}$, the equation that conforms to the least square principle need to be established,

$$
Q=\sum_{i=0}^{11} \sum_{j=0}^{11} V_{i, j}{ }^{2}=\min \quad, i \neq j
$$

If the partial derivative of the function $Q$ to the unknown is 0 ,

$$
\frac{\partial Q}{\partial \delta \alpha_{i}}=0, \quad i=1,2, \cdots, 11
$$

The deviation of the angle comparator's angles can be calculated by the following equations

$$
\delta \alpha_{i}=\frac{t_{i}-t_{0}}{2 * 12}
$$

where,

$$
\begin{aligned}
& t_{i}=q_{i}-p_{i} \\
& q_{i}=\sum_{j=0}^{11} \theta_{j, i}, \quad i \neq j \\
& p_{i}=\sum_{j=0}^{11} \theta_{i, j}, \quad i \neq j
\end{aligned}
$$

\section{Results}

\subsection{Experiment results}

The deviations of nominal angles of a 1440 precision indexing table were measured using the angle comparator, the optical mirror and the calibrated autocollimator aforementioned in Sec. 3. In the experiment, we applied the complete closure method to obtain the deviation angle of the indexing table and the error of the angle comparator separately. 


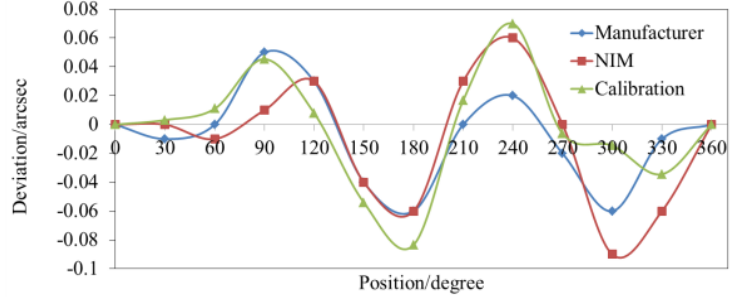

Figure 6. Comparison of the calibration results of the indexing table.

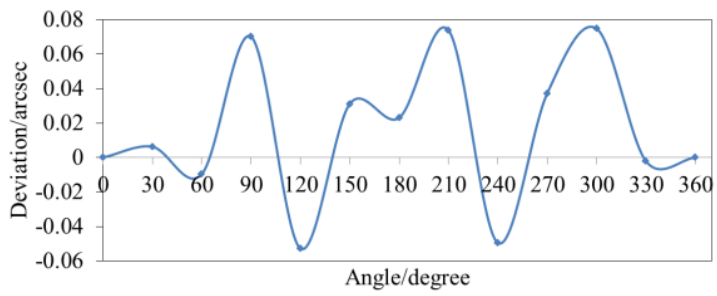

Figure 7. The position error of the angle comparator by the complete closure method.

The measurement result of the indexing table was compared with the calibration result provided by the manufacturer and the National Institute of Metrology of China (NIM). The comparison data curve is shown in Figure 6 . The expanded uncertainty $(\mathrm{k}=2)$ reported in the calibration certificate of the manufacturer is $0.05 "$, and that of the NIM is also $0.05 "$. These calibration results show good agreement considering the magnitude of each uncertainty.

Figure 7 shows the position error of the angle comparator by the complete closure method, the error of the angle comparator was evaluated to be about $0.08 "$.

\subsection{Uncertainty analysis}

The combined measurement uncertainty $u_{c}$, attributed to the indexing table calibration is determined in accordance with the Guide to the Expression of Uncertainty in Measurement (GUM) [10] by the addition of several single standard uncertainties that are important for the measuring process. According to the Eq. (7), the propagation formula can be deduced as:

$$
u_{c}^{2}=\sum_{i=1}^{m} c_{i}^{2} u_{i}^{2}
$$

where the sensitive coefficient of every component $c_{i}$ is $1, u_{i}$ is a relevant standard uncertainty.

Table 1 shows a list of all relevant uncertainty components for the calibration of indexing table using a high precision angle comparator and states the estimates, the distribution function and the resulting uncertainty value as standard uncertainly. Measurement uncertainty contributions are explained in following.

Table 1. Uncertainty budget for the calibration of indexing table.

\begin{tabular}{|c|l|c|c|c|c|}
\hline Type & \multicolumn{1}{|c|}{ Uncertainty component } & $\begin{array}{c}\text { Estimate } \\
\text { /arcsec }\end{array}$ & Distribution function & $\begin{array}{c}\text { sensitive } \\
\text { coeff. } c_{i}\end{array}$ & $\begin{array}{c}\text { Uncertainty contribution/ } \\
\operatorname{arcsec} u_{i}\end{array}$ \\
\hline A & $\begin{array}{l}\text { Standard deviation of the mean } \\
\text { value }\end{array}$ & & Normal & 0.017 \\
\hline B & $\begin{array}{l}\text { Measurement repeatability of the } \\
\text { angle comparator }\end{array}$ & 0.02 & Normal & 0.0067 \\
\hline B & $\begin{array}{l}\text { Resolution of the angle } \\
\text { comparator }\end{array}$ & 0.0017 & Rectangular & \multirow{2}{*}{0} \\
\hline B & Resolution of the autocollimator & 0.005 & Rectangular & 0.0005 \\
\hline B & $\begin{array}{l}\text { Repeatability of the test indexing } \\
\text { table }\end{array}$ & 0.02 & Rectangular & 0.0014 \\
\hline B & $\begin{array}{l}\text { Uncertainty of the angular } \\
\text { reflector }\end{array}$ & 0.012 & Rectangular & 0.0058 \\
\hline
\end{tabular}

Table 2. Calculating data of measurement repeatability.

\begin{tabular}{|c|c|c|c|c|c|c|c|c|c|c|c|c|}
\hline Position & $0^{\circ}$ & $30^{\circ}$ & $60^{\circ}$ & $90^{\circ}$ & $120^{\circ}$ & $150^{\circ}$ & $180^{\circ}$ & $210^{\circ}$ & $240^{\circ}$ & $270^{\circ}$ & $300^{\circ}$ & $330^{\circ}$ \\
\hline$\alpha_{\mathrm{i}}$ & 0.00 & 0.00 & 0.11 & 0.08 & 0.01 & 0.12 & 0.13 & 0.13 & 0.09 & 0.12 & 0.15 & 0.08 \\
\hline$\alpha_{\mathrm{i}}^{\prime}$ & 0.00 & 0.00 & 0.10 & 0.11 & 0.07 & 0.18 & 0.16 & 0.17 & 0.12 & 0.15 & 0.18 & 0.13 \\
\hline
\end{tabular}

\subsubsection{The uncertainty contribution of autocollimator standard deviation of the mean value $u 1$}

During the calibration, standard deviation of the mean value produced from 10 series of repeat measurements and the maximal standard measurement deviation of 10 measurements is $0.055 "$. According to the principle of the standard values merge, the uncertainty u1 is 0.017 ".

\subsubsection{The uncertainty contribution of measurement repeatability of the angle comparator $\mathrm{U}_{2}$}

The measurement repeatability of the angle comparator is obtained by double-observation method, that is, measurement is performed at 12 positions evenly distributed in the whole circumference. Table 2 shows the calculating data of measurement repeatability. 
The measurement repeatability is

$$
u_{2}=\frac{1}{3} s_{r}=\frac{1}{3} \sqrt{\frac{\sum_{i=1}^{n}\left(\alpha_{i}-\alpha_{i}^{\prime}\right)^{2}}{2 n}}=0.0067^{\prime \prime}
$$

\subsubsection{The uncertainty contribution of resolution of the angle comparator $u_{3}$}

The resolution of the angle comparator is 0.0017" resulting in $u_{3}=0.0017 /(2 \times \sqrt{3})=0.0005^{\prime \prime} \quad$ uncertainty contribution.

\subsubsection{The uncertainty contribution of resolution of the autocollimator $u_{4}$}

The resolution of the autocollimator is 0.005 " resulting in $u_{4}=0.005 /(2 \times \sqrt{3})=0.0014^{\prime \prime}$ uncertainty contribution.

\subsubsection{The uncertainty contribution of repeatability of the test indexing table 45}

The repeatability of the test indexing table provided by the manufacturer is 0.02 resulting in $u_{5}=0.02 /(2 \times \sqrt{3})=0.0058^{\prime \prime}$ uncertainty contribution.

\subsubsection{The uncertainty contribution of the angular reflector $u 6$}

During the calibration, the optical polygon, whose working surface is used as an angular reflector, and autocollimator are used together, the flatness of the working surface is $50 \mathrm{~nm}$ in its central area, the dimension of the working surface is $12 \mathrm{~mm} \times 15 \mathrm{~mm}$. When the autocollimator aims at different position of the reflector, the flatness will have influence on the angle measurement.

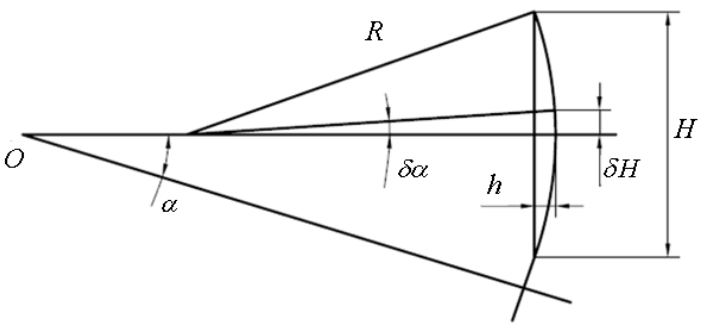

Figure 8. Illustration of the uncertainty contribution of the angular reflector

Assuming that the surface of the reflector is approximately arc shape, the curvature radius of the reflector is

$$
R=\frac{4 h^{2}+H^{2}}{8 h} \approx \frac{H^{2}}{8 h}
$$

where $\mathrm{h}$ is the flatness of the reflector, $\mathrm{H}$ is the width of the reflector.
When the autocollimator optical axis deviates from working face center at a distance of $\delta H$, it will cause the normal angle of working face to produce angle deviation $\delta \alpha$,

$$
\delta \alpha=\frac{\delta H}{R} \rho=\frac{8 h \delta H}{H^{2}} \rho
$$

where $\delta H$ is $0.02 \mathrm{~mm}, \rho$ is $206265^{\prime \prime} / \mathrm{rad}$.

So, the uncertainty contribution of the angular reflector is

$$
u_{6}=\frac{\delta \alpha}{\sqrt{3}}=\frac{8 h \delta H}{\sqrt{3} H^{2}} \rho=0.007^{\prime \prime}
$$

\subsubsection{The expanded measurement uncertainty $U$}

The expanded measurement uncertainty $U$ is calculated by multiplying the combined standard uncertainty, uc , by the coverage factor $k=2$. The value of the calibration results thus lie with a probability of $95 \%$ within the attributed interval of values.

$$
U=k \sqrt{\sum_{i=1}^{m} c_{i}^{2} u_{i}^{2}}=0.04^{\prime \prime}
$$

\section{Conclusions}

A high precision angle comparator is developed in this research. It uses four ultrasonic motors and a double readheads encoder adopting a virtual gantry control mode to produce fine driving resolution and high accuracy with simple configuration. To calibrate the indexing table, a measurement system including the optical polygon, the angle comparator, and the autocollimator was built up, and the mathematical model of complete closure method is established. Applying the complete closure method to the calibration of the indexing table, both the angle comparator's position error and the indexing table's angular deviation are obtained separately based on the least square method. To verify the performance of the angle generator, the indexing table is measured and the experimental results are analyzed. Through the calibration, the position error of the angle comparator is evaluated to be about 0.08 ". The calibration result of the indexing table agreed with the calibration data from both the manufacturer and National Institute of Metrology of China within the calibration uncertainty, 0.04 " . Through the experiment, the performance of the angle generator is proved.

\section{Acknowledgment}

This work was supported by Science and Technology Major Project of China Academy of Engineering Physics: (9120602), Calibration and Testing Lab Project of China Academy of Engineering Physics: (16S006).

\section{References}


1. A. Just, M. Krause, R. Probst, et al. Comparison of angle standards with the aid of high-resolution angle encoder. Precision Engineering, 33, 4(2009): 530-533

2. Ralf D Geckeler, Andreas Fricke, Clemens Elster, Calibration of angle encoders using transfer functions. Measurement Science and Technology, 17, 10(2006): 2811-2818

3. W.T. Estler, Y.H. Queen, J. Bryan. Advanced angle metrology system. CIRP Annals, 42, 1(1993): 573576

4. Tanfer Yandayan, S Asli Akgoz, Muharrem Asar. Calibration of high-resolution electronic autocollimators with demanded low uncertainties using single reading head angle encoders. Measurement Science and Technology, 25, 1(2014): 015010

5. A Just, M Krause, R Probst, et al. Calibration of high-resolution electronic autocollimators against an angle comparator. Metrologia, 40, 5(2003): 288-294

6. Jong-Ahn Kim, Jae Wan Kim, Chu-Shik Kang, et al. Precision angle comparator using self-calibration of scale errors based on the equal-division-averaged method. Proc. of MacroScale, (2011)

7. Jong-Ahn Kim, Jae Wan Kim, Chu-Shik Kang, et al. High precision angle generator using multiple ultrasonic motors and a self-calibratable encoder. Review of Scientific Instruments, 82, 11(2011): 116108-1-116108-3

8. Jong-Ahn Kim, Jae Wan Kim, Chu-Shik Kang, et al. Calibration of angle artifacts and instruments using a high precision angle generator. International Journal Of Precision Engineering And Manufacturing, 14, 3(2013): 367-371

9. Yao Huang, Zi Xue, Heyan Wang. Comparison between angle interferometer and angle encoder during calibration of autocollimator. Ninth International Symposium on Precision Engineering Measurement and Instrumentation. 9446, (2015): 944624

10. ISO/IEC. Uncertainty of measurement: part 3. Guide to the Expression of Uncertainty in Measurement. ISO/IEC Guide 98-3, (2008) 